\title{
The Supplement Adulterant $\beta$-Methylphenethylamine Increases Blood Pressure by Acting at Peripheral Norepinephrine Transporters
}

\author{
Charles W. Schindler, Eric B. Thorndike, Kenner C. Rice, John S. Partilla, \\ and Michael H. Baumann
}

Designer Drug Research Unit (C.W.S., J.S.P., M.H.B.) and Preclinical Pharmacology Section (E.B.T.), National Institute on Drug Abuse Intramural Research Program, Baltimore, Maryland; and Drug Design and Synthesis Section, National Institute on Drug Abuse and National Institute of Alcohol Abuse and Alcoholism Intramural Research Programs, Rockville, Maryland (K.C.R.)

Received December 18, 2018; accepted March 18, 2019

\begin{abstract}
$\beta$-Methylphenethylamine [(BMPEA), 2-phenylpropan-1-amine] is a structural isomer of amphetamine (1-phenylpropan-2-amine) that has been identified in preworkout and weight loss supplements, yet little information is available about its pharmacology. Here, the neurochemical and cardiovascular effects of BMPEA and its analogs, $N$-methyl-2-phenylpropan-1-amine (MPPA) and $N, N$-dimethyl-2-phenylpropan-1-amine (DMPPA), were compared with structurally related amphetamines. As expected, amphetamine and methamphetamine were potent substrate-type releasing agents at dopamine transporters (DATs) and norepinephrine transporters (NETs) in rat brain synaptosomes. BMPEA and MPPA were also substrates at DATs and NETs, but they were at least 10 -fold less potent than amphetamine. DMPPA was a weak substrate only at NETs. Importantly, the releasing actions of BMPEA and MPPA were more potent at NETs than DATs.
\end{abstract}

Amphetamine produced significant dose-related increases in blood pressure $(\mathrm{BP})$, heart rate $(\mathrm{HR})$, and locomotor activity in conscious rats fitted with surgically implanted biotelemetry transmitters. BMPEA, MPPA, and DMPPA produced increases in BP that were similar to the effects of amphetamine, but the compounds failed to substantially affect HR or activity. The hypertensive effect of BMPEA was reversed by the $\alpha$-adrenergic antagonist prazosin but not the ganglionic blocker chlorisondamine. Radioligand binding at various $\mathrm{G}$ protein-coupled receptors did not identify nontransporter sites of action that could account for cardiovascular effects of BMPEA or its analogs. Our results show that BMPEA, MPPA, and DMPPA are biologically active. The compounds are unlikely to be abused due to weak effects at DATs, but they could produce adverse cardiovascular effects via substrate activity at peripheral NET sites.

\section{Introduction}

Dietary supplements are used by a significant portion of the population, and many supplement products contain ingredients that are not properly listed on the label. The phenomenon of unlisted ingredients is well established for supplements that are promoted for body building and performance enhancement (i.e., preworkout supplements) (Eichner et al., 2016; Rasmussen and Keizers, 2016). As a specific example, preworkout supplements that list the Southwestern scrub plant Acacia rigidula as an ingredient often contain $\beta$-methylphenethylamine [(BMPEA, 2-phenylpropan-1-amine], a structural isomer of amphetamine (1-phenylpropan-2-amine). BMPEA was first described in the $1930 \mathrm{~s}$ as a sympathomimetic agent (Cohen et al., 2016). While the manufacturers of preworkout

This research was supported by the Intramural Research Program of the National Institutes of Health National Institute on Drug Abuse and National Institute of Alcohol Abuse and Alcoholism (Z1ADA000523, Z1ADA000532).

https://doi.org/10.1124/jpet.118.255976. supplements claim that Acacia rigidula is a natural source of BMPEA, there is no evidence to support this assertion (Cohen et al., 2015). Thus, the US Food and Drug Administration (FDA) concluded that products containing BMPEA were adulterated and banned their sale in 2015 (Pawar and Grundel, 2017). Despite the fact that BMPEA and related adulterants are banned by the FDA, chemical analogs often still appear in supplement products (Eichner et al., 2016). Many of these compounds have not been fully tested for safety, and as a result individuals using these supplements may be at risk for serious medical complications (Venhuis et al., 2014).

Cohen et al. (2015) reported the case of a woman who ingested a sports supplement 30 minutes before exercise and subsequently experienced "numbness and clumsiness in her left hand." The patient reported taking no other drugs prior to exercise. A computed tomography scan revealed she had a "2-cm hemorrhage in the right parietal lobe." Forensic analysis of the ingested supplement determined that it contained BMPEA at a level of $290 \mathrm{mg}$ when used at the recommended

ABBREVIATIONS: BMPEA, $\beta$-methylphenethylamine (2-phenylpropan-1-amine); BP, blood pressure; DAT, dopamine transporter; DMPPA, $N$, $N$-dimethyl-2-phenylpropan-1-amine; $E_{\max }$, maximum release; FDA, Food and Drug Administration; GBR12909, 1-[2-[bis-(4-fluorophenyl)methoxy]ethyl]4-(3-phenylpropyl)piperazine; GPCR, G protein-coupled receptor; HR, heart rate; 5-HT, 5-hydroxytryptamine; MPP ${ }^{+}$, methyl-4-phenylpyridinium; MPPA, Nmethyl-2-phenylpropan-1-amine; NET, norepinephrine transporter; SERT, serotonin transporter. 
amount. While the pharmacology of BMPEA has not been investigated in humans, older studies from around the time of its original synthesis show it can increase blood pressure (BP) in dogs (Graham et al., 1945; Marsh, 1948; Winder et al., 1948), rabbits (Hartung and Munch, 1931; Warren et al., 1943), and rats (Graham and Cartland, 1944). Therefore, it seems feasible that hypertension following the ingestion of the supplement containing BMPEA contributed to the occurrence of hemorrhagic stroke described by Cohen et al. (2015).

As noted previously, the FDA banned the addition of BMPEA to supplements in 2015, but BMPEA and related compounds are still found in certain supplement products (Cohen et al., 2016; Rasmussen and Keizers, 2016). Because these compounds are structurally related to amphetamine, it seems possible that they have pharmacological and toxic effects similar to amphetamine and other stimulants. Here, we studied the pharmacology of BMPEA and its secondary [ $N$-methyl-2-phenylpropan-1-amine (MPPA)] and tertiary [N,N-dimethyl-2-phenylpropan-1-amine (DMPPA)] amine analogs (see Fig. 1 for chemical structures). For comparison, we also investigated amphetamine and its secondary ( $N$-methyl1-phenylpropan-2-amine, methamphetamine) and tertiary ( $N, N$-dimethyl-1-phenylpropan-2-amine, dimethylamphetamine) amine analogs. All compounds were first evaluated for substrate-type releasing activity at dopamine transporters (DATs), norepinephrine transporters (NETs), and serotonin transporters (SERTs) using in vitro functional assays in rat brain synaptosomes. BMPEA and its analogs were also tested for binding affinity at various $G$ protein-coupled receptors (GPCRs) using in vitro assays in cells transfected with human receptors. Finally, the in vivo effects of systemically administered BMPEA and its analogs were examined in rats fitted with surgically implanted biotelemetry transmitters to measure BP, heart rate (HR), temperature, and locomotor activity.

\section{Materials and Methods}

All procedures reported here were approved by the National Institute on Drug Abuse Intramural Research Program Animal Care and Use Committee and followed the guidelines described in the
Guide for the Care and Use of Laboratory Animals (National Research Council, 2011). Animals were housed in facilities fully accredited by the Association for the Assessment and Accreditation of Laboratory Animal Care.

Drugs and Reagents. The $\beta$-methyl compounds BMPEA and MPPA were synthesized as fumarate salts, whereas DMPPA was synthesized as $\mathrm{HCl}$ salt. BMPEA, MPPA, and DMPPA were synthesized using standard organic chemical reactions and techniques. Each compound was fully characterized by high-resolution mass spectral and $400 \mathrm{MHz}$ NMR analyses and gave appropriate combustion analyses for carbon, hydrogen, and nitrogen. Each compound was chromatographically homogenous by thin-layer chromatography. Chemical purity for each compound was estimated to be greater than $98 \%$. The corresponding $\alpha$-methyl comparator compounds $(S)$ amphetamine sulfate (amphetamine), $(S)$-methamphetamine $\mathrm{HCl}$ (methamphetamine), and racemic $N, N$-dimethylamphetamine $\mathrm{HCl}$ (dimethylamphetamine) were obtained from the National Institute on Drug Abuse Intramural Research Program Pharmacy in Baltimore, MD. $\left[{ }^{3} \mathrm{H}\right]$ Methyl-4-phenylpyridinium [ $\left.\left(\mathrm{MPP}^{+}\right) ; 80 \mathrm{Ci} / \mathrm{mmol}\right]$ was purchased from American Radiolabeled Chemicals (St. Louis, MO), while $\left[{ }^{3} \mathrm{H}\right] 5$ - hydroxytryptamine [(5-HT), $\left.38 \mathrm{Ci} / \mathrm{mmol}\right]$ was purchased from Perkin Elmer (Billerica, MA). All other chemicals and reagents were acquired from Sigma-Aldrich (St Louis, MO) unless otherwise noted.

In Vitro Transporter Release Assays in Synaptosomes. Adult male Sprague-Dawley rats weighing 300-400 g (Charles River Laboratories, Kingston, NY) were used for the synaptosome assays. Rats were group-housed in a temperature $\left(22.2 \pm 1.1^{\circ} \mathrm{C}\right)$ and humidity $(45 \% \pm 10 \%)$ controlled room under a standard 12-hour light/dark cycle (lights on at 7:00 AM), with free access to food and water. Rats were euthanized by $\mathrm{CO}_{2}$ narcosis, synaptosomes were prepared from brain tissue, and transporter release assays were performed as described previously (Rothman et al., 2001; Baumann et al., 2013; Solis et al., 2017). In brief, synaptosomes were prepared from caudate tissue for DAT assays, whereas synaptosomes were prepared from whole brain minus caudate and cerebellum for NET and SERT assays. For release assays, $9 \mathrm{nM}\left[{ }^{3} \mathrm{H}\right] \mathrm{MPP}^{+}$was used as the radiolabeled substrate for DATs and NETs, while $5 \mathrm{nM}\left[{ }^{3} \mathrm{H}\right] 5-\mathrm{HT}$ was used for SERTs. All buffers used in the release assay contained $1 \mu \mathrm{M}$ reserpine to block vesicular uptake of substrates. The selectivity of release assays was optimized for a single transporter by including unlabeled blockers to prevent the uptake of $\left[{ }^{3} \mathrm{H}\right] \mathrm{MPP}^{+}$or $\left[{ }^{3} \mathrm{H}\right] 5$-HT by competing transporters. Synaptosomes were preloaded with radiolabeled substrate in Krebs phosphate buffer for 1 hour to achieve steady state. Release assays were initiated by incubating preloaded synaptosomes<smiles>CC(N)Cc1ccccc1</smiles>

Amphetamine<smiles>CNC(C)Cc1ccccc1</smiles>

Methamphetamine<smiles>CC(Cc1ccccc1)N(C)C</smiles><smiles>CC(CN(C)C)c1ccccc1</smiles>

$N, N$-Dimethyl-2-phenylpropan1-amine (DMPPA)<smiles>CC(CN)c1ccccc1</smiles><smiles>CNCC(C)c1ccccc1</smiles>

Fig. 1. Chemical structures of amphetamine and BMPEA analogs examined in this study. 
with various concentrations of test drugs. Release was terminated by vacuum filtration, and retained radioactivity was quantified by liquid scintillation counting.

To verify that drug-induced release of $\left[{ }^{3} \mathrm{H}\right] \mathrm{MPP}^{+}$from synaptosomes was mediated by the DAT or NET, we conducted substrate reversal experiments. For substrate reversal studies, synaptosome release assays were conducted as described previously in the presence or absence of a fixed concentration of 1-[2-[bis-(4-fluorophenyl)methoxy]ethyl]-4(3-phenylpropyl)piperazine [(GBR12909), $1 \mathrm{nM}]$ or desipramine ( $8 \mathrm{nM})$ to block the DAT or NET, respectively. Previous findings demonstrated that coincubation of uptake blockers with releasers at the DAT or NET will produce parallel rightward shifts in the release curves for true transporter substrates (Rothman et al., 2001). Effects of test drugs on release were expressed as percentage of maximum release $\left(E_{\max }\right)$, i.e., $100 \% E_{\max }$, with $E_{\max }$ defined as the release produced by tyramine at doses that evoke the efflux of all releasable tritium by synaptosomes (10 $\mu \mathrm{M}$ tyramine for DAT and NET assay conditions, and $100 \mu \mathrm{M}$ tyramine for SERT assay conditions). Effects of test drugs on release were analyzed by nonlinear regression using GraphPad Prism version 6 (GraphPad Scientific, San Diego, CA), with dose-response data fit to the equation $Y(x)=Y_{\min }+\left(Y_{\max }-Y_{\min }\right) /$ $\left[1+10 \exp \left(\log P_{50}-\log x\right) \times n\right]$, where $x$ is the concentration of the compound tested, $Y(x)$ is the response measured, $Y_{\max }$ is the maximal response, $P_{50}$ is the $\mathrm{EC}_{50}$ (the concentration that yields half-maximal release), and $n$ is the Hill slope parameter.

In Vitro Receptorome Screening in Transfected Cells. BMPEA and its analogs were submitted to the Psychoactive Drug Screening Program of the National Institute on Mental Health and evaluated for binding activity at various human GPCRs transfected in cells, according to established protocols (Besnard et al., 2012). In particular, the activity of test drugs at receptor subtypes for dopamine, norepinephrine, 5-HT, histamine, and opioids was examined. Compounds were first screened at a fixed concentration of $10 \mu \mathrm{M}$ to assess inhibition of receptor binding. In those instances where binding was inhibited by more than $50 \%$ at $10 \mu \mathrm{M}$, full dose-response curves were obtained, and $K_{\mathrm{i}}$ values (in nanomolars) were calculated by nonlinear regression using the Cheng-Prusoff equation.

In Vivo Biotelemetry in Rats. A total of 12 adult male SpragueDawley rats weighing 300-400 g (Charles River Laboratories) were used as subjects. Rats were purchased by Data Sciences International (St. Paul, MN) and subsequently received surgically implanted intraperitoneal HD-S10 biotelemetry transmitters to measure changes in BP, HR, activity, and temperature. Surgery was carried out by experienced technicians at Data Sciences International. For the surgery, rats were anesthetized with isoflurane and the abdominal cavity opened. The descending aorta was isolated, and the catheter from the transmitter was inserted into the aorta and glued in place. The abdominal muscles and skin were then closed. Rats were treated with subcutaneous meloxicam following surgery. After recovery from surgery at Data Sciences International, the rats were shipped to the National Institute on Drug Abuse Intramural Research Program in Baltimore, MD, and underwent 7-day quarantine.

Following release from quarantine, the rats were individually housed in a temperature $\left(22.2 \pm 1.1^{\circ} \mathrm{C}\right)$ and humidity $(45 \% \pm 10 \%)$ controlled room under a 12 -hour reverse light-dark cycle (lights off at 7:00 AM) with free access to water. Food was restricted to maintain a constant or slowly increasing weight of approximately $400-500$ g over the course of the experiments. Rats were subsequently adapted to the experimental chambers and injection procedure over a period of 3 to 4 weeks. Each weekday the rats were transported to the procedure room, where food and water were removed from the home cage and the entire home cage was placed on top of a telemetry receiver (RPC-001; Data Sciences) inside a small acoustical chamber (BRS/LVE, Laurel, MD). Transmitters were turned on by placing a magnet near the abdomen of the rat. The chambers were then closed, and experimental parameters were monitored for 3 hours. At the end of the session, the transmitters were turned off by again placing a magnet near the abdomen of the rats, food and water were replaced to the home cage, and the rats were returned to the housing room. Once experimental parameters were stable from day to day, injections of saline were given subcutaneously at least twice per week (typically, on Tuesdays and Fridays) 5 minutes prior to the rats being placed in the experimental chamber. Once experimental parameters in response to saline injection were again stable, experimental procedures with test drugs commenced.

Dose-effect determinations for BMPEA (3-30 $\mathrm{mg} / \mathrm{kg})$, MPPA (3-30 mg/kg), DMPPA (3-30 mg/kg), and amphetamine $(0.1-3 \mathrm{mg} / \mathrm{kg})$ were determined in seven rats. These rats had previously been tested with combinations of 3,4-methylenedioxypyrovalerone and chlorisondamine, prazosin, or propranolol for prior experiments (Schindler et al., 2016); some rats had also received synthetic cannabinoids (Schindler et al., 2017) or $\alpha$-pyrrolidinovalerophenone. Sufficient washout time of greater than 4 weeks was allowed between prior testing and current testing to avoid drug interactions. Order for the dose-effect testing was nonsystematic, although all rats were typically tested with the same drug and dose on any given test day. All drugs were administered subcutaneously 5 minutes prior to placement of the rat in the experimental chamber. Saline was tested every 2 to 3 weeks and responses following saline were stable over the testing period.

Five additional rats were tested with combinations of prazosin $(0.3 \mathrm{mg} / \mathrm{kg})$ or chlorisondamine $(1 \mathrm{mg} / \mathrm{kg})$ and BMPEA $(30 \mathrm{mg} / \mathrm{kg})$. These rats had previously been tested with $\alpha$-ethyl-phenethylamine. As with dose-effect testing, a minimum 4-week washout period was allowed for the effects of $\alpha$-ethyl-phenethylamine to dissipate and not interact with current testing. Order of testing was identical to that described for dose-effect testing. Prazosin was administered subcutaneously 5 minutes prior to BMPEA, which was given 5 minutes prior to placement in the chamber. Chlorisondamine was given subcutaneously 10 minutes prior to BMPEA. Appropriate controls for pretreatment alone and vehicle alone were tested for both prazosin and chlorisondamine. The effects of $30 \mathrm{mg} / \mathrm{kg}$ BMPEA alone were similar to the separate group of rats in the dose-effect group. In addition, $30 \mathrm{mg} / \mathrm{kg}$ BMPEA was tested twice in the pretreatment group about 4 months apart and produced similar results in each test, indicating that there were no long-term effects of the drugs that could alter the results.

Data from biotelemetry transmitters were collected for 10 -second epochs every minute and these 1-minute readings were averaged over the first hour for statistical analysis. The transmitters supplied readings for $\mathrm{BP}$ (i.e., mean arterial pressure), HR (derived from the BP signal), temperature, and motor activity. Motor activity was measured by tracking the strength of the transmitter radio signal as the rat moved about the home cage; therefore, these measures do not have any units. Dose-effect data for each drug were subjected to ANOVA (Prism version 6), followed by Dunnett's multiple comparisons test, which can compare drug effects to control. Data for the drug interaction studies were also subject to ANOVA, followed by Tukey's multiple comparisons test, which allows for comparisons between all groups tested.

\section{Results}

In Vitro Transporter Release Assays in Synaptosomes. Figure 2 shows dose-effect curves for drug-induced efflux (i.e., release) of $\left[{ }^{3} \mathrm{H}\right] \mathrm{MPP}^{+}$at DATs (upper panels) and NETs (lower panels), with the calculated $\mathrm{EC}_{50}$ and $\% E_{\max }$ values shown in Table 1. As expected, amphetamine and methamphetamine were potent substrate-type releasers at both DATs and NETs. Dimethylamphetamine was a much less potent releaser than amphetamine at DATs and somewhat less potent at NETs, leading to a preference for NETs over DATs. It is noteworthy that dimethylamphetamine displayed partial releasing activity at the DAT, reaching only $78 \%$ of $E_{\max }$ values. Amphetamine and methamphetamine 


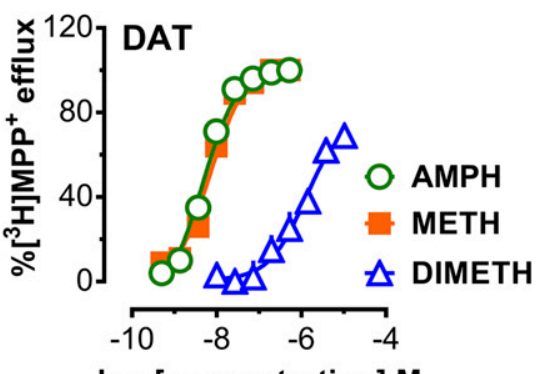

log [concentration] M

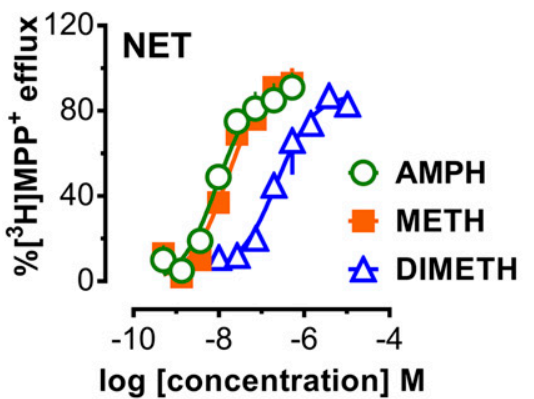

log [concentration] M
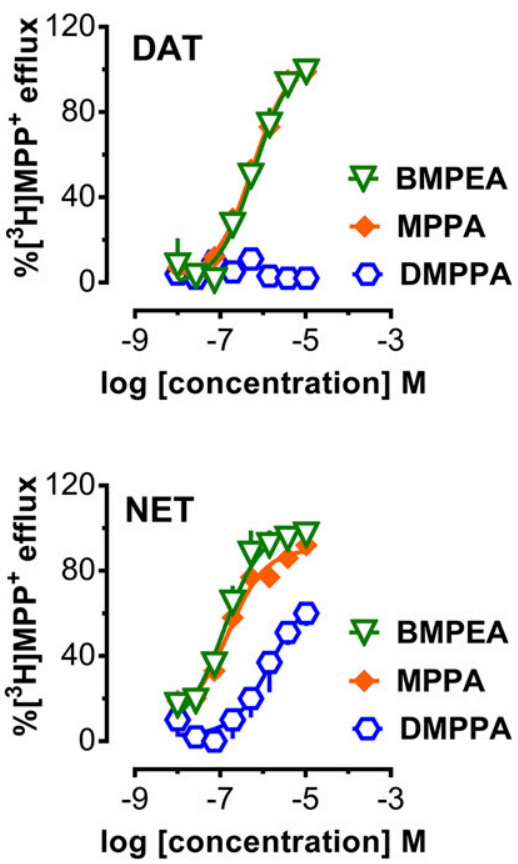

Fig. 2. Concentration-response effects for analogs of amphetamine (AMPH) and BMPEA to stimulate $\left[{ }^{3} \mathrm{H}\right]$ $\mathrm{MPP}^{+}$efflux (i.e., release) via the DAT (upper panels) or NET (lower panels). Rat brain synaptosomes were preloaded with $9 \mathrm{nM}\left[{ }^{3} \mathrm{H}\right] \mathrm{MPP}^{+}$, and different concentrations of AMPH, methamphetamine (METH), dimethylamphetamine (DIMETH), BMPEA, MPPA, or DMPPA were added to stimulate release. Results are plotted as a percentage of maximal release elicited by $10 \mu \mathrm{M}$ tyramine. Data are mean \pm S.D. for $N=3$ separate experiments performed in triplicate. were weak releasers at the SERT, while dimethylamphetamine was inactive at this transporter at concentrations up to $10 \mu \mathrm{M}$ (data not shown). BMPEA and MPPA produced fullefficacy release at DATs but were $>50$-fold less potent when compared with amphetamine and methamphetamine in this regard. DMPPA was completely inactive as a substrate at the DAT. BMPEA and MPPA were full-efficacy releasers at the NET, while DMPPA was a weak partial releaser (i.e., 67\% $\left.E_{\text {max }}\right)$ at this transporter. BMPEA, MPPA, and DMPPA were about 10 -fold less potent than amphetamine and its analogs as substrates at the NET. It is noteworthy that BMPEA, MPPA, and DMPPA showed a clear preference for NETs over DATs, and none of the compounds were substrates at SERTs at concentrations up to $10 \mu \mathrm{M}$.

Figure 3 shows the effects of an uptake inhibitor at the DAT (GBR12909) or NET (desipramine) on amphetamine- and BMPEA-induced release of $\left[{ }^{3} \mathrm{H}\right] \mathrm{MPP}^{+}$from rat brain synaptosomes. Coincubation with GBR12909 produced a parallel rightward shift in the DAT-mediated release curves for amphetamine (6.2-fold increase in $\mathrm{EC}_{50}$ ) and BMPEA (4.7-fold increase in $\mathrm{EC}_{50}$ ). GBR12909 had a comparable effect on the releasing activity of MPPA (4.6-fold increase in $\left.\mathrm{EC}_{50}\right)$. The findings with GBR12909 indicate that amphetamine, BMPEA, and MPPA exert their releasing actions at DATs by interacting competitively with a binding site blocked by GBR12909. In a similar manner, coincubation with desipramine shifted the NET-mediated release curves for both amphetamine (3.8-fold increase in $\mathrm{EC}_{50}$ ) and BMPEA (5.7-fold increase in $\mathrm{EC}_{50}$ ). Desipramine also produced a rightward shift in the release curves for MPPA (4.1-fold shift) and DMPPA (4.4-fold shift) (data not shown). The results with desipramine indicate that amphetamine, BMPEA, and its analogs exert their releasing actions at NETs by interacting competitively with a binding site blocked by desipramine.

In Vitro Receptorome Screening in Transfected Cells. Table 2 presents the results for BMPEA, MPPA, and DMPPA in the GPCR screening in comparison with amphetamine. In general, BMPEA and its analogs had little activity at GPCRs when tested at a $10 \mu \mathrm{M}$ concentration. Specific exceptions included the $5-\mathrm{HT}_{1 \mathrm{~A}}$ receptor, where BMPEA and MPPA had mid-nanomolar affinities (375-915 nM); alpha2 receptor subtypes, where BMPEA showed affinities in the range of its transporter releasing potency (288-1739 $\mathrm{nM})$; and the sigma-1 receptor, where MPPA and DMPPA showed low

TABLE 1

Effects of amphetamine and BMPEA and their respective analogs on release of $\left[{ }^{3} \mathrm{H}\right] \mathrm{MPP}^{+}$at the DAT and NET in rat brain synaptosomes

Data are mean \pm S.D. for $N=3$ experiments performed in triplicate. The $\% E_{\text {max }}$ value is defined as the percentage of maximal releasing response induced by $10 \mu \mathrm{M}$ tyramine. DAT/NET ratio $=\left(\mathrm{DAT} \mathrm{IC}_{50}{ }^{-1}\right) /\left(\mathrm{NET} \mathrm{IC}_{50}{ }^{-1}\right)$; higher values indicate greater DAT selectivity.

\begin{tabular}{lccc}
\hline \multicolumn{1}{c}{ Drug } & Release at $\mathrm{DAT} \mathrm{EC}_{50}\left[\% E_{\max }\right]$ & Release at NET EC $\mathrm{Fo}_{50}\left[\% E_{\max }\right]$ & DAT/NET Ratio \\
\hline & $n M$ & $n M$ & 1.80 \\
Amphetamine & $5 \pm 1[104]$ & $9 \pm 2[95]$ & 2.29 \\
Methamphetamine & $7 \pm 2[108]$ & $16 \pm 3[97]$ & 0.18 \\
Dimethylamphetamine & $1250 \pm 231[78]$ & $223 \pm 40[84]$ & 0.20 \\
BMPEA & $627 \pm 102[101]$ & $126 \pm 21[94]$ & 0.27 \\
MPPA & $574 \pm 52[103]$ & $154 \pm 25[80]$ & - \\
DMPPA & Inactive & $1337 \pm 354[67]$ & - \\
\hline
\end{tabular}




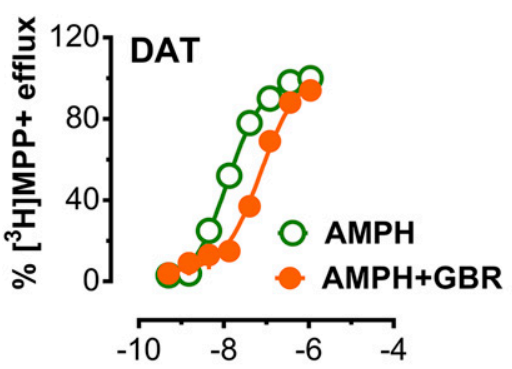

log [concentration] M

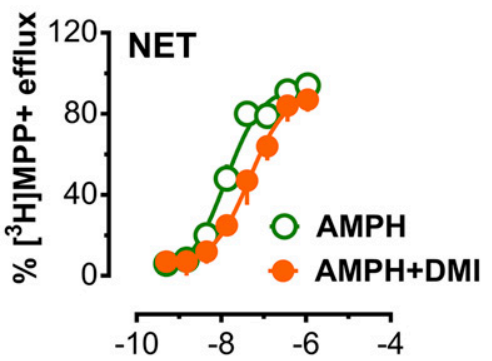

log [concentration] M
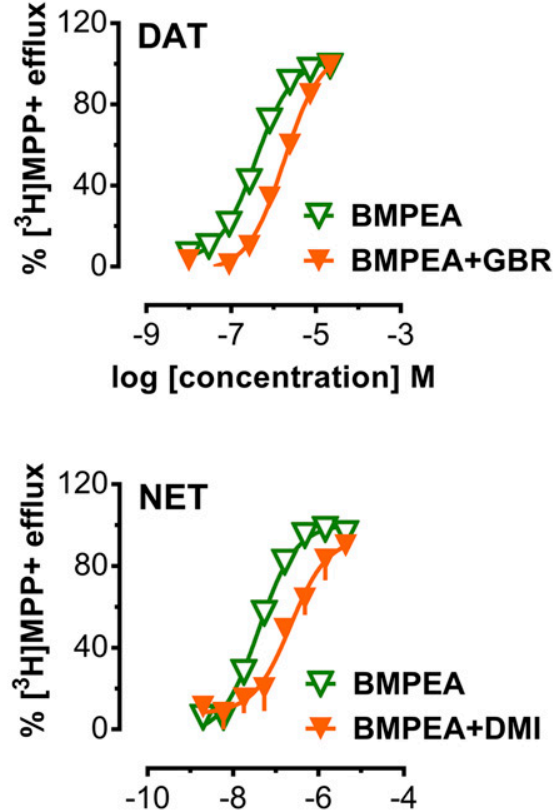

$\log$ [concentration] M
Fig. 3. Concentration-response effects for amphetamine (AMPH) and BMPEA to stimulate $\left[{ }^{3} \mathrm{H}\right] \mathrm{MPP}^{+}$ efflux (i.e., release) in the presence or absence of the DAT blocker GBR12909 [(GBR), upper panels] or the NET blocker desipramine [(DMI), lower panels]. Rat brain synaptosomes were preloaded with $9 \mathrm{nM}\left[{ }^{3} \mathrm{H}\right]$ $\mathrm{MPP}^{+}$, and different concentrations of AMPH or BMPEA were added to stimulate release in the presence or absence of $1 \mathrm{nM} \mathrm{GBR} 12909$ for DAT assays or $8 \mathrm{nM}$ desipramine for NET assays. Data are mean \pm S.D. for $N=3$ separate experiments performed in triplicate. micromolar affinities. BMPEA and its analogs also had low micromolar affinities to inhibit binding to the sigma-2 site. Interestingly, none of the BMPEA analogs potently inhibited binding to DATs, NETs, or SERTs. While this finding seems

\section{TABLE 2}

G protein-coupled receptor screening for amphetamine and BMPEA and their analogs

Data represent $K_{\mathrm{i}}$ values obtained from nonlinear regression using the Cheng-Prusoff equation when inhibition of binding at $10 \mu \mathrm{M}$ was above $50 \%$.

\begin{tabular}{|c|c|c|c|c|}
\hline Receptor Family & AMPH & BMPEA & MPPA & DMPPA \\
\hline & $n M$ & $n M$ & $n M$ & $n M$ \\
\hline \multicolumn{5}{|l|}{ Serotonin } \\
\hline $5-\mathrm{HT}_{1 \mathrm{~A}}$ & 2625 & 375 & 915 & $>10,000$ \\
\hline $5-\mathrm{HT}_{1 \mathrm{~B}}$ & $>10,000$ & $>10,000$ & $>10,000$ & $>10,000$ \\
\hline $5-\mathrm{HT}_{1 \mathrm{D}}$ & $>10,000$ & $>10,000$ & $>10,000$ & $>10,000$ \\
\hline $5-\mathrm{HT}_{1 \mathrm{E}}$ & $>10,000$ & $>10,000$ & $>10,000$ & $>10,000$ \\
\hline $5-\mathrm{HT}_{2 \mathrm{~A}}$ & $>10,000$ & $>10,000$ & $>10,000$ & $>10,000$ \\
\hline $5-\mathrm{HT}_{2 \mathrm{~B}}$ & 971 & $>10,000$ & $>10,000$ & $>10,000$ \\
\hline $5-\mathrm{HT}_{2 \mathrm{C}}$ & $>10,000$ & $>10,000$ & $>10,000$ & $>10,000$ \\
\hline SERT & $>10,000$ & $>10,000$ & $>10,000$ & $>10,000$ \\
\hline \multicolumn{5}{|l|}{ Norepinephrine } \\
\hline Alpha1A & $>10,000$ & $>10,000$ & $>10,000$ & $>10,000$ \\
\hline Alpha1B & $>10,000$ & $>10,000$ & $>10,000$ & $>10,000$ \\
\hline Alpha1D & $>10,000$ & $>10,000$ & $>10,000$ & $>10,000$ \\
\hline Alpha2A & 420 & 1739 & $>10,000$ & $>10,000$ \\
\hline Alpha2B & 192 & 288 & $>10,000$ & $>10,000$ \\
\hline Alpha2C & 171 & 962 & $>10,000$ & $>10,000$ \\
\hline Beta1 & $>10,000$ & $>10,000$ & $>10,000$ & $>10,000$ \\
\hline Beta2 & $>10,000$ & $>10,000$ & $>10,000$ & $>10,000$ \\
\hline Beta3 & $>10,000$ & $>10,000$ & $>10,000$ & $>10,000$ \\
\hline NET & 31 & $>10,000$ & $>10,000$ & $>10,000$ \\
\hline \multicolumn{5}{|l|}{ Dopamine } \\
\hline D1 & $>10,000$ & $>10,000$ & $>10,000$ & $>10,000$ \\
\hline D2 & $>10,000$ & $>10,000$ & $>10,000$ & $>10,000$ \\
\hline D3 & $>10,000$ & $>10,000$ & $>10,000$ & $>10,000$ \\
\hline D4 & $>10,000$ & $>10,000$ & $>10,000$ & $>10,000$ \\
\hline D5 & $>10,000$ & $>10,000$ & $>10,000$ & $>10,000$ \\
\hline DAT & $>10,000$ & $>10,000$ & $>10,000$ & $>10,000$ \\
\hline \multicolumn{5}{|l|}{ Sigma } \\
\hline Sigma 1 & $>10,000$ & $>10,000$ & 1233 & 1636 \\
\hline Sigma 2 & $>10,000$ & 2735 & 8110 & 2802 \\
\hline
\end{tabular}

counterintuitive, previous studies have demonstrated that amphetamine and other monoamine transporter substrates display very weak ability to displace high-affinity ligands in binding assays for DATs, NETs, and SERTs (Eshleman et al., 1999, 2017; Rothman et al., 1999).

In Vivo Biotelemetry in Rats. In general, the rats with surgically implanted telemetry transmitters adapted quickly to the experimental procedure, and results for the control conditions remained stable throughout testing. Importantly, there was no indication that previous drug treatments altered the baseline for any of the parameters reported in the present study. Following subcutaneous vehicle injections, BP and HR values were slightly elevated immediately after placement of the rats into the experimental chamber but declined over the first 60 minutes and remained steady until the end of the session (Fig. 4).

Figure 4 shows the time course for the effects of amphetamine and BMPEA on BP (upper panels) and HR (lower panels) in conscious rats. Amphetamine produced dosedependent increases in both parameters that were sustained throughout the 3-hour session. BMPEA also produced a dosedependent increase in BP, but the effects dissipated throughout the session and by the end of the 3 hours had returned to the saline control level. The effects of BMPEA on HR were more complicated, with small increases observed at the lower doses and the highest dose producing a substantial decrease. Again, the effects of BMPEA were mostly restricted to the first hour of the session. Because the most prominent effects of BMPEA were in the beginning of the session, the analysis of mean effects presented below is based only on the first hour of measurements.

As depicted in Fig. 5, amphetamine produced significant dose-dependent increases in mean $\mathrm{BP}\left(F_{4,34}=20.9 ; P<0.001\right)$ and $\operatorname{HR}\left(F_{4,34}=11.1 ; P<0.001\right)$. Amphetamine also produced significant increases in motor activity $\left(F_{4,34}=9.4 ; P<0.001\right)$, although this effect peaked at $1 \mathrm{mg} / \mathrm{kg}$ and the higher dose failed to produce a significant increase in activity. This 

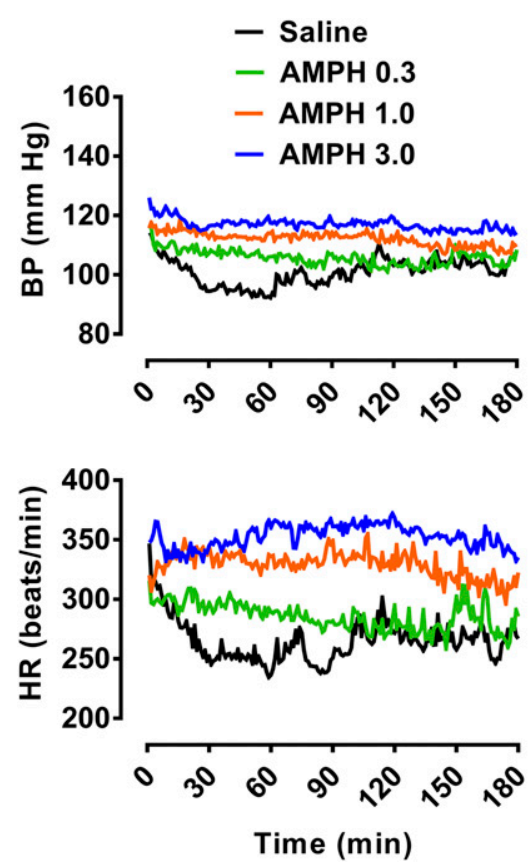
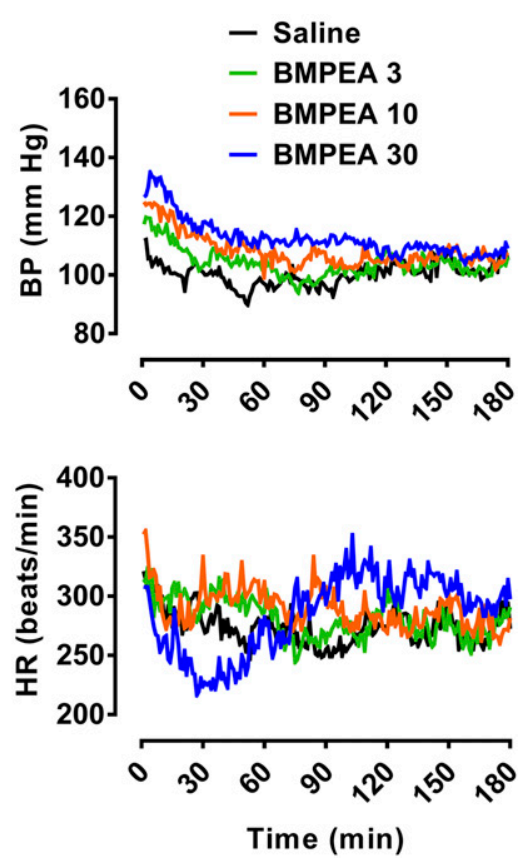

Fig. 4. Time-course effects of amphetamine (AMPH) and BMPEA to influence BP (upper panels) and HR (lower panels) in conscious rats bearing biotelemetry transmitters. Rats received subcutaneous injections of AMPH or BMPEA and were subsequently moved to recording chambers for 3 -hour test sessions. Mean values for $N=7$ rats/group are plotted for 1-minute epochs over the entire 180-minute sampling period. decrease in motor activity at the highest amphetamine dose could be due to the tendency for this drug, and other psychomotor stimulants, to produce in-place stereotypy at higher doses that the telemetry system does not detect. Amphetamine did not produce significant changes in temperature.

While less potent than amphetamine, BMPEA also dosedependently increased BP $\left(F_{3,27}=15.0 ; P<0.001\right)$. MPPA produced hypertensive effects similar to, or even greater than, BMPEA $\left(F_{3,27}=20.8 ; P<0.001\right)$. While DMPPA also tended to increase BP compared with saline control, this effect failed to reach significance. In contrast to the clear dose-dependent effects of amphetamine on HR, the cardiac effects of the other compounds were less uniform. BMPEA slightly decreased $\mathrm{HR}$ at the highest dose $\left(F_{3,27}=6.7\right.$; $P<0.01$ ), while MPPA slightly increased $\mathrm{HR}$ at the two lowest doses $\left(F_{3,27}=5.5 ; P<0.01\right)$. DMPPA did not significantly affect HR. In striking contrast to amphetamine, none of the other drugs tested affected motor activity. BMPEA slightly decreased body temperature at the two highest doses $\left(F_{3,27}=8.9 ; P<0.001\right)$. To summarize, all four drugs produced significant and dose-dependent increases in BP. However, in contrast to the robust effects of amphetamine on HR and activity, BMPEA and its analogs produced small variable effects on $\mathrm{HR}$ and no significant stimulation of motor activity. Only BMPEA affected body temperature, with a small decrease that was restricted to the first hour of the session.
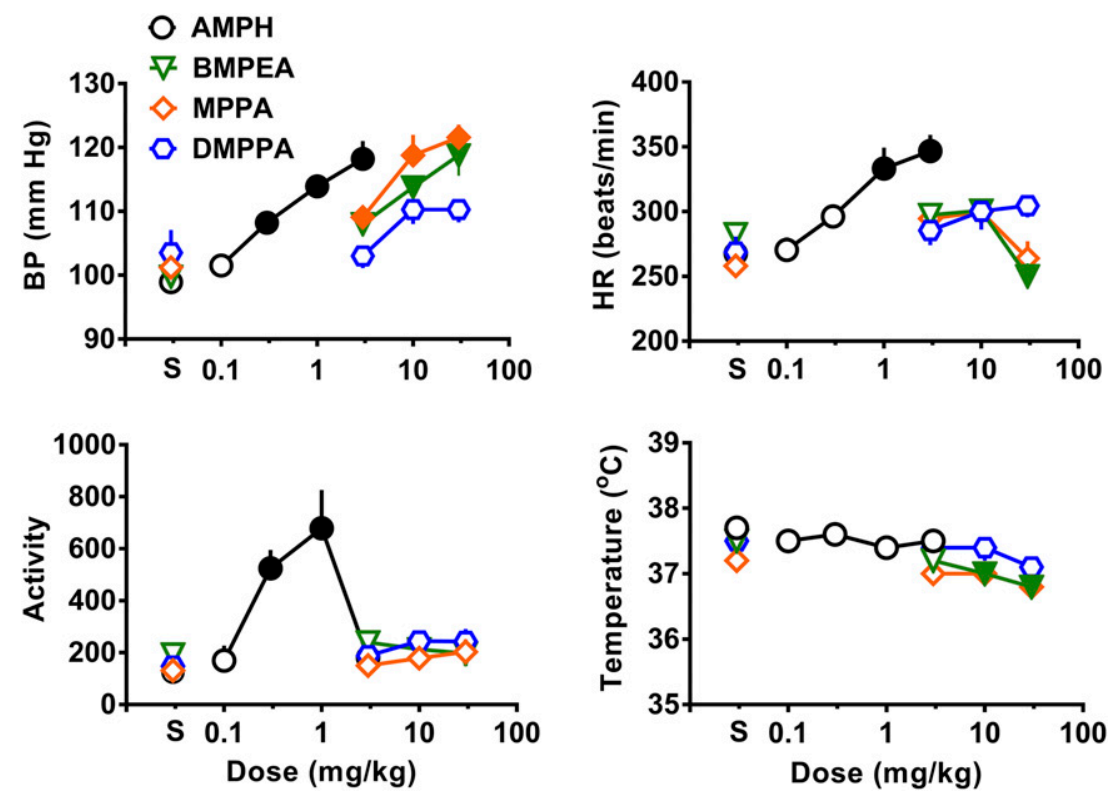

Fig. 5. Dose-effect functions for amphetamine (AMPH), BMPEA, MPPA, and DMPPA on BP, HR, motor activity, and body temperature. Data are mean values from the first hour of test sessions, where the effects of the test drugs were maximal. Solid symbols indicate significant differences compared with the respective saline control group. Data are mean \pm S.E.M. for $N=7 \mathrm{rats} /$ group. 
Because BMPEA is frequently encountered in preworkout and weight loss supplements, and its most prominent effect was on the cardiovascular system, we next determined whether the ganglionic blocker chlorisondamine or the $\alpha$-adrenergic antagonist prazosin could antagonize those effects. Figure 6 shows the effects of pretreatment with $1 \mathrm{mg} / \mathrm{kg}$ chlorisondamine or $0.3 \mathrm{mg} / \mathrm{kg}$ prazosin on $\mathrm{BP}$ (upper panels) and HR (lower panels) changes induced by $30 \mathrm{mg} / \mathrm{kg}$ BMPEA. As in the dose-effect determination, $30 \mathrm{mg} / \mathrm{kg}$ BMPEA significantly increased BP $\left(F_{3,19}=114.6\right.$ and $P<0.001$ in the chlorisondamine graph; $F_{3,19}=32.9$ and $P<0.001$ in the prazosin graph). The hypertensive effect of BMPEA was not antagonized by chlorisondamine, even though chlorisondamine alone decreased $\mathrm{BP}$, suggesting a peripheral site of action for BMPEA. BMPEA alone produced a small decrease in HR for the chlorisondamine tests $\left(F_{3,19}=\right.$ 99.83; $P<0.001$ ); however, when pretreated with chlorisondamine, that HR decrease was converted to significant tachycardia, even though chlorisondamine on its own had no effects on HR. The hypertensive effects of BMPEA were completely antagonized by prazosin, indicating the $\mathrm{BP}$ increase is mediated via the sympathetic nervous system. For HR, prazosin alone produced tachycardia $\left(F_{3,19}=31.76 ; P<0.01\right)$. When administered after prazosin, BMPEA increased HR over saline; however, this effect was no different than prazosin alone, which complicated interpretation of the result.

\section{Discussion}

BMPEA and related compounds continue to be detected as adulterants in preworkout and weight loss supplements, despite being banned by the FDA (Cohen et al., 2016; Eichner et al., 2016; Rasmussen and Keizers, 2016). The main objective of the present work was to examine the pharmacology of BMPEA and its $N$-methylated analogs, MPPA and DMPPA. Because BMPEA is a structural isomer of amphetamine, we reasoned that the compounds might share similar mechanisms of action and pharmacological profiles in vivo. Findings from our in vitro transporter release assays confirm that amphetamine and methamphetamine are potent substrate-type releasers at DATs and NETs, with much weaker activity at SERTs (Rothman et al., 2001; Rothman and Baumann, 2003). Similar to amphetamine, BMPEA also acted as a substrate-type releaser at DATs and NETs, but showed a clear preference for NETs over DATs. In contrast to amphetamine, BMPEA was inactive in the SERT release assay at concentrations up to $10 \mu \mathrm{M}$. For both amphetamine and BMPEA, coincubation with the dopamine uptake inhibitor GBR12909 shifted the dose-effect curve for DATmediated $\left[{ }^{3} \mathrm{H}\right] \mathrm{MPP}^{+}$efflux to the right in a parallel manner (i.e., increased $\mathrm{EC}_{50}$ values), indicating a decrease in releasing potency. Likewise, the norepinephrine uptake inhibitor desipramine shifted the dose-effect curve for NET-mediated release to the right. Data from the substrate reversal experiments provide compelling evidence that BMPEA and its analogs interact with the orthosteric binding site on DATs and NETs to exert their neurotransmitter releasing actions, analogous to the mechanism of action for amphetamine and its analogs (Rothman and Baumann, 2003; Reith et al., 2015; Sitte and Freissmuth, 2015).

BMPEA failed to inhibit radioligand binding to DATs or NETs, and this observation is consistent with prior studies demonstrating that substrate-type releasing drugs display weak ability to displace high-affinity ligands in transporter binding assays (Rothman et al., 1999; Simmler et al., 2013; Eshleman et al., 2017). However, the present NET binding results illustrate an important difference between amphetamine and BMPEA. Previous studies have shown that amphetamine displays a $K_{\mathrm{i}}$ value of $\sim 4 \mu \mathrm{M}$ for NET sites labeled with the cocaine analog $\left[{ }^{125} \mathrm{I}\right]$ methyl $(1 R, 2 S, 3 S)-3-(4-$ iodophenyl)-8-methyl-8-azabicyclo[3.2.1]octane-2-carboxylate (Eshleman et al., 1999), whereas we report a $K_{\mathrm{i}}$ value of $30 \mathrm{nM}$
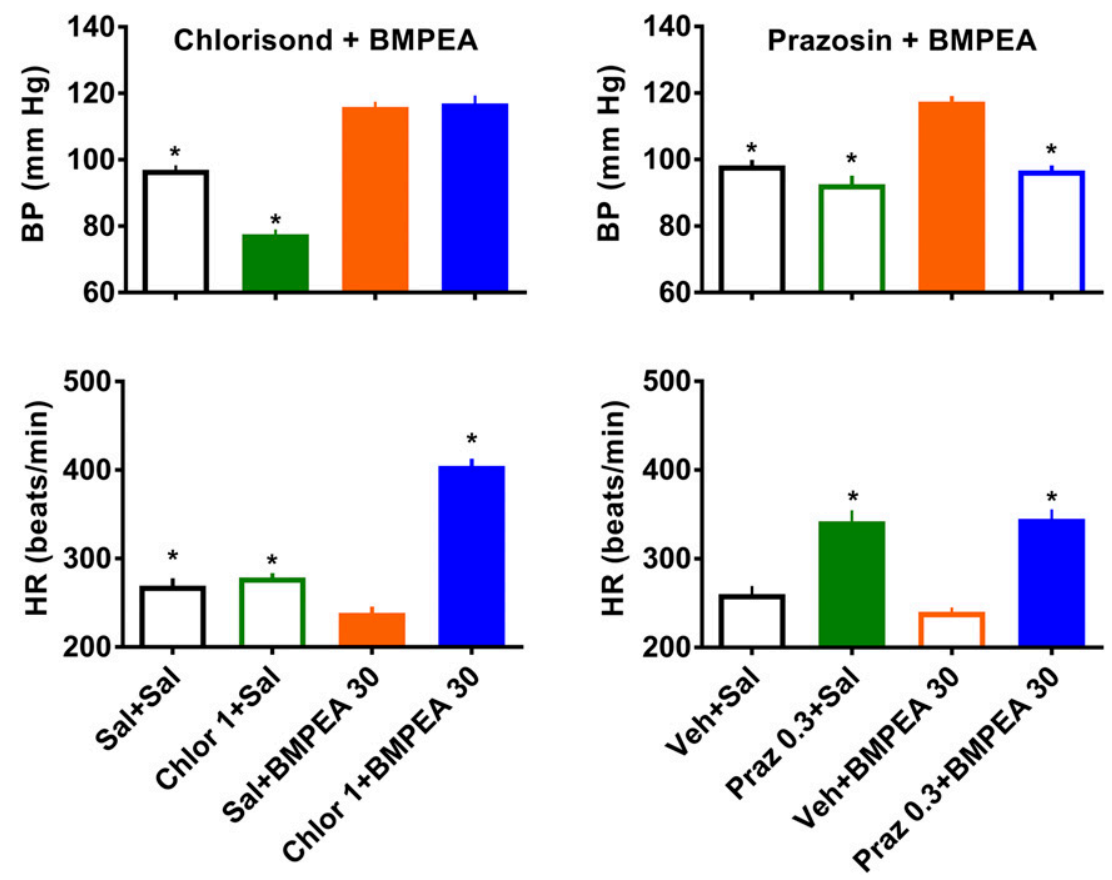

Fig. 6. Effects of pretreatment with the ganglionic blocker chlorisondamine $(1 \mathrm{mg} / \mathrm{kg}$, s.c.) or the $\alpha 1$ adrenoreceptor antagonist prazosin $(0.3 \mathrm{mg} / \mathrm{kg}$, s.c.) on BP (upper panels) or HR (lower panels) produced by $30 \mathrm{mg} / \mathrm{kg}$ BMPEA. Data are mean \pm S.E.M. from the first hour of the sessions, where the effects of BMPEA were maximal; $N=5$ rats/group. Filled bars represent a significant difference from saline + saline $(\mathrm{Sal}+\mathrm{Sal})$; * indicates a significant difference from BMPEA alone [saline (Sal) or vehicle (Veh) + BMPEA 30]. 
for NET sites labeled with $\left[{ }^{3} \mathrm{H}\right]$ nisoxetine. Thus, the $\left[{ }^{3} \mathrm{H}\right]$ nisoxetine binding assay uncovers a substantial affinity difference between amphetamine and BMPEA that is not mirrored in the release assays. One interpretation of these data is that amphetamine and BMPEA might interact with the NET protein in a somewhat different manner to exert their effects, and future studies should investigate this possibility.

From a structure-activity perspective, we found that adding steric bulk to the terminal amine of amphetamine or BMPEA produced notable changes in functional measures of transporter activity. For example, adding a single methyl group to amphetamine (i.e., methamphetamine) had minimal influence on releasing potency at DATs or NETs, while adding a second methyl group (i.e., dimethylamphetamine) markedly decreased releasing potency in both assays. Likewise, for BMPEA, adding one methyl group to the terminal amine (i.e., MPPA) had minimal effect on releasing potency at DATs or NETs. However, adding a second methyl group (i.e., DMPPA) produced a dramatic decrease in releasing potency at NETs and a complete loss of releasing activity at DATs. The present findings with BMPEA and its $N$-methylated analogs are generally consistent with previous results that show $N$-ethyl derivatives of amphetamines or $\beta$-keto amphetamines (i.e., cathinones) display substrate activity at NETs but markedly reduced or absent substrate activity at DATs (Saha et al., 2015; Solis et al., 2017). The preference of BMPEA and its analogs for NETs over DATs suggests that these compounds may not be abused to a significant degree. It is well established that the abuse potential of stimulant drugs is related to their ability to increase extracellular concentrations of dopamine in the brain (Rothman and Baumann, 2003; Sitte and Freissmuth, 2015; Volkow et al., 2017). On the other hand, the preference of BMPEA and its analogs for NETs infers a risk for cardiovascular side effects similar to those produced by amphetamines.

In our telemetry experiments, amphetamine produced clear dose-dependent and sustained increases in BP and HR, in agreement with previous results (Broadley, 2010; Varner et al., 2013). BMPEA and MPPA produced robust dosedependent increases in BP similar in magnitude to the effects of amphetamine but were about 10 -fold less potent than amphetamine. While trending in the direction of an increase, the hypertensive effect of DMPPA was not statistically significant. In contrast to amphetamine, the effects of BMPEA and its analogs on HR were complex. BMPEA produced a significant, albeit small, decrease in HR at the highest dose, while lower doses tended to increase HR. MPPA slightly increased HR at lower doses, while DMPPA did not. It is noteworthy that cardiovascular perturbations produced by BMPEA, MPPA, and DMPPA were brief when compared with effects of amphetamine, suggesting that $\beta$-methyl compounds are metabolized or eliminated rapidly after systemic administration. Pretreatment with the ganglionic blocker chlorisondamine did not reverse the hypertensive effect of BMPEA, despite the fact that chlorisondamine alone produced hypotension. This result demonstrates that the effects of BMPEA on BP do not involve a central mechanism but are mediated peripherally. Further supporting this conclusion was the observation that chlorisondamine pretreatment converted BMPEA-induced bradycardia to marked tachycardia. This intriguing observation suggests BMPEA actually produces a peripherally mediated increase in $\mathrm{HR}$ that is normally counteracted by a centrally mediated baroreceptor reflex to decrease HR. Finally, the $\alpha 1$-adrenoreceptor antagonist prazosin blocked the hypertensive effects of BMPEA, indicating this response involves activation of the sympathetic nervous system. Taken together with the findings in synaptosomes, the telemetry results suggest that BMPEA and its analogs increase BP by a mechanism involving transporter-mediated release of norepinephrine from peripheral stores, which then activates $\alpha 1$-adrenoreceptors in vascular tissue.

Extrapolation of preclinical results from rats to humans is always problematic due to a number of different factors. For example, we administered BMPEA to rats by the subcutaneous route, whereas humans take BMPEA by the oral route. Nevertheless, controlled clinical studies demonstrate that $20 \mathrm{mg}$ of oral amphetamine (i.e., $0.29 \mathrm{mg} / \mathrm{kg}$ for a $70 \mathrm{~kg}$ subject) increases BP in human volunteers (Sofuoglu et al., 2009; Wardle and de Wit, 2012), and we show similar effects after $0.3 \mathrm{mg} / \mathrm{kg}$ of subcutaneous amphetamine in rats (Figs. 4 and 5). Such observations indicate that our in vivo preclinical data might be directly translatable to humans. With regard to BMPEA, we found this compound to be 10 -fold less potent than amphetamine in its ability to increase BP in rats. Given that $290 \mathrm{mg}$ of BMPEA is a reported oral dose in humans (Cohen et al., 2015), it seems feasible that this amount of BMPEA could elevate BP. Future clinical studies are warranted to investigate the cardiovascular effects of BMPEA and related compounds.

It is important to consider the possibility that BMPEA, MPPA, and DMPPA might act at nontransporter sites of action, most notably GPCRs, to produce their effects on BP. Assuming these drugs are acting at the same site to produce their effects on BP, then one can assume that their rank order of potency at a given site of action would parallel their order of potency to produce hypertension $($ BMPEA $=$ MPPA $>$ DMPPA). BMPEA showed weaker affinity than DMPPA at the sigma-1 receptor, which likely rules out this site as a potential target. At the sigma-2 receptor, BMPEA and DMPPA were equipotent but displayed lower $K_{\mathrm{i}}$ values than MPPA, again suggesting this site is not involved with hypertensive effects of BMPEA and its analogs. BMPEA had mid-nanomolar affinities at subtypes of $\alpha 2$-adrenoreceptors, and these sites can modulate BP (c.f. van Zwieten, 1999). However, the fact that MPPA had equal or greater effects on BP than BMPEA, but was inactive at $\alpha 2$-adrenoreceptor subtypes, appears to rule out a role for these sites in the BP changes observed. Finally, BMPEA and MPPA had similar affinity for the $5-\mathrm{HT}_{1 \mathrm{~A}}$ receptor. Actions at the $5-\mathrm{HT}_{1 \mathrm{~A}}$ receptor can also influence BP (Anderson et al., 1992; Ramage and Villalón, 2008), but DMPPA had virtually no affinity for $5-\mathrm{HT}_{1 \mathrm{~A}}$ receptors yet still produced increases in BP. Therefore, it seems reasonable to assume that BMPEA and its analogs produce increases in BP via a mechanism that relies on peripheral NET sites rather than direct actions at GPCRs.

We found that amphetamine produced dramatic increases in locomotor activity, a characteristic of abused psychomotor stimulants (Rothman and Baumann, 2003; Sitte and Freissmuth, 2015). The highest dose of amphetamine did not produce an increase in locomotor activity, which likely reflects an increase in focused in-place stereotypies at this dose. Although not measured directly here, an increase in stereotypy at higher doses is typical of psychomotor stimulants (c.f. Wolgin, 2012), and the small repetitive movements 
associated with stereotypy may not be detected by the telemetry system used here. BMPEA and its analogs failed to produce significant changes in motor activity. This result may be related to the relatively weak effects of these compounds at DATs compared with NETs, but may also reflect poor penetration of the compounds across the bloodbrain barrier. The fact that these compounds do not increase motor activity like most stimulant drugs of abuse supports the aforementioned contention that BMPEA, MPPA, and DMPPA may not exhibit significant abuse potential. Neither amphetamine nor BMPEA analogs had robust effects on body temperature at doses tested here. Only BMPEA produced a significant decrease in body temperature, and this effect was only $0.5^{\circ} \mathrm{C}$, well within the normal variance of body temperature under physiologic conditions. Thus, it appears that these compounds do not substantially affect core body temperature regulation, at least when the animals are kept at room temperature.

In summary, the findings presented here demonstrate that BMPEA and its analogs produce transient dose-dependent increases in BP that resemble the effects of amphetamine. Converging lines of evidence indicate that hypertensive effects are mediated through the peripheral sympathetic nervous system, most likely by substrate-type releasing actions at NETs. BMPEA and related compounds show a distinct preference for releasing activity at NETs over DATs, and do not produce motor activation, suggesting the compounds will have minimal abuse potential. On the other hand, since these analogs are still being found in preworkout and weight loss supplements that are not routinely tested for safety (Cohen et al., 2016; Eichner et al., 2016; Rasmussen and Keizers, 2016), the risk for cardiovascular side effects remains a possibility in vulnerable individuals. Future studies should examine the pharmacology and toxicology of other amphetaminerelated compounds that are present as adulterants in supplements (e.g., $N, \alpha$-diethylphenethylamine), since consumers are being unknowingly exposed to these substances.

\section{Authorship Contributions}

Participated in research design: Schindler, Baumann.

Conducted experiments: Schindler, Thorndike, Partilla, Baumann. Contributed new reagents or analytic tools: Rice.

Performed data analysis: Schindler, Thorndike, Baumann.

Wrote or contributed to the writing of the manuscript: Schindler, Rice, Baumann.

\section{References}

Anderson IK, Martin GR, and Ramage AG (1992) Central administration of 5-HT activates 5-HT1A receptors to cause sympathoexcitation and 5-HT2/5-HT1C receptors to release vasopressin in anaesthetized rats. $\mathrm{Br} J$ Pharmacol 107: $1020-1028$.

Baumann MH, Partilla JS, Lehner KR, Thorndike EB, Hoffman AF, Holy M, Rothman RB, Goldberg SR, Lupica CR, Sitte HH, et al. (2013) Powerful cocaine-like actions of 3,4-methylenedioxypyrovalerone (MDPV), a principal constituent of psychoactive 'bath salts' products. Neuropsychopharmacology 38:552-562.

Besnard J, Ruda GF, Setola V, Abecassis K, Rodriguiz RM, Huang XP, Norval S, Sassano MF, Shin AI, Webster LA, et al. (2012) Automated design of ligands to polypharmacological profiles. Nature 492:215-220.

Broadley KJ (2010) The vascular effects of trace amines and amphetamines. Pharmacol Ther 125:363-375.

Cohen PA, Bloszies C, Yee C, and Gerona R (2016) An amphetamine isomer whose efficacy and safety in humans has never been studied, $\beta$-methylphenylethylamine (BMPEA), is found in multiple dietary supplements. Drug Test Anal 8:328-333.

Cohen PA, Zeijlon R, Nardin R, Keizers PH, and Venhuis B (2015) Hemorrhagic stroke probably caused by exercise combined with a sports supplement containing $\beta$-methylphenyl-ethylamine (BMPEA): a case report. Ann Intern Med 162:879-880.

Eichner S, Maguire M, Shea LA, and Fete MG (2016) Banned and discouraged-use ingredients found in weight loss supplements. J Am Pharm Assoc (2003) 56 538-543.
Eshleman AJ, Carmolli M, Cumbay M, Martens CR, Neve KA, and Janowsky A (1999) Characteristics of drug interactions with recombinant biogenic amine transporters expressed in the same cell type. J Pharmacol Exp Ther 289 877-885.

Eshleman AJ, Wolfrum KM, Reed JF, Kim SO, Swanson T, Johnson RA, and Janowsky A (2017) Structure-activity relationships of substituted cathinones, with transporter binding, uptake, and release. J Pharmacol Exp Ther 360:33-47.

Graham BE and Cartland GF (1944) Some comparative pharmacological actions of beta-hydroxy and methoxy phenyl-n-propylamines. J Pharmacol Exp Ther 81: 360-367.

Graham BE, Cartland GF, and Woodruff EH (1945). Phenyl propyl and phenyl isopropyl amines. Ind Eng Chem 37:149-151.

Hartung WH and Munch JC (1931) The preparation and pharmacodynamic activity of four isomeric phenylpropylamines. J Am Chem Soc 53:1875-1879.

Marsh DF (1948) The comparative pharmacology of the cyclohexylalkylamines. $J$ Pharmacol Exp Ther 93:338-345.

National Research Council (2011) Guide for the Care and Use of Laboratory Animals, 8th ed, The National Academies Press, Washington, DC.

Pawar RS and Grundel E (2017) Overview of regulation of dietary supplements in the USA and issues of adulteration with phenethylamines (PEAs). Drug Test Anal 9: 500-517.

Ramage AG and Villalón CM (2008) 5-Hydroxytryptamine and cardiovascular regulation. Trends Pharmacol Sci 29:472-481.

Rasmussen N and Keizers PH (2016) History full circle: 'Novel' sympathomimetics in supplements. Drug Test Anal 8:283-286.

Reith ME, Blough BE, Hong WC, Jones KT, Schmitt KC, Baumann MH, Partilla JS, Rothman RB, and Katz JL (2015) Behavioral, biological, and chemical perspectives on atypical agents targeting the dopamine transporter. Drug Alcohol Depend 147: $1-19$

Rothman RB, Ayestas MA, Dersch CM, and Baumann MH (1999) Aminorex, fenfluramine, and chlorphentermine are serotonin transporter substrates. Implications for primary pulmonary hypertension. Circulation 100:869-875.

Rothman RB and Baumann MH (2003) Monoamine transporters and psychostimulant drugs. Eur $J$ Pharmacol 479:23-40.

Rothman RB, Baumann MH, Dersch CM, Romero DV, Rice KC, Carroll FI, and Partilla JS (2001) Amphetamine-type central nervous system stimulants release norepinephrine more potently than they release dopamine and serotonin. Synapse 39:32-41.

Saha K, Partilla JS, Lehner KR, Seddik A, Stockner T, Holy M, Sandtner W, Ecker GF, Sitte HH, and Baumann MH (2015) 'Second-generation' mephedrone analogs, 4-MEC and 4-MePPP, differentially affect monoamine transporter function. Neuropsychopharmacology 40:1321-1331.

Schindler CW, Gramling BR, Justinova Z, Thorndike EB, and Baumann MH (2017) Synthetic cannabinoids found in "spice" products alter body temperature and cardiovascular parameters in conscious male rats. Drug Alcohol Depend 179: 387-394.

Schindler CW, Thorndike EB, Suzuki M, Rice KC, and Baumann MH (2016) Pharmacological mechanisms underlying the cardiovascular effects of the "bath salt" constituent 3,4-methylenedioxypyrovalerone (MDPV). $\mathrm{Br} J$ Pharmacol 173 3492-3501.

Simmler LD, Buser TA, Donzelli M, Schramm Y, Dieu LH, Huwyler J, Chaboz S, Hoener MC, and Liechti ME (2013) Pharmacological characterization of designer cathinones in vitro. Br J Pharmacol 168:458-470.

Sitte HH and Freissmuth M (2015) Amphetamines, new psychoactive drugs and the monoamine transporter cycle. Trends Pharmacol Sci 36:41-50.

Sofuoglu M, Poling J, Hill K, and Kosten T (2009) Atomoxetine attenuates dextroamphetamine effects in humans. Am J Drug Alcohol Abuse 35:412-416.

Solis E Jr, Partilla JS, Sakloth F, Ruchala I, Schwienteck KL, De Felice LJ, Eltit JM, Glennon RA, Negus SS, and Baumann MH (2017) $N$-Alkylated analogs of 4-methylamphetamine (4-MA) differentially affect monoamine transporters and abuse liability. Neuropsychopharmacology 42:1950-1961.

van Zwieten PA (1999) Centrally acting antihypertensive drugs. Present and future. Clin Exp Hypertens 21:859-873.

Varner KJ, Daigle K, Weed PF, Lewis PB, Mahne SE, Sankaranarayanan A, and Winsauer PJ (2013) Comparison of the behavioral and cardiovascular effects of mephedrone with other drugs of abuse in rats. Psychopharmacology (Berl) 225: 675-685.

Venhuis B, Keizers P, van Riel A, and de Kaste D (2014) A cocktail of synthetic stimulants found in a dietary supplement associated with serious adverse events. Drug Test Anal 6:578-581.

Volkow ND, Wise RA, and Baler R (2017) The dopamine motive system: implications for drug and food addiction. Nat Rev Neurosci 18:741-752.

Wardle MC and de Wit H (2012) Effects of amphetamine on reactivity to emotional stimuli. Psychopharmacology (Berl) 220:143-153.

Warren MR, Marsh DG, Thompson CR, Shelton RS, and Becker TJ (1943) Pharmacological studies on d,l-B-phenyl-n-propylmethylamine, a volatile amine. $J$ Pharmacol Exp Ther 79:187-199.

Winder CV, Anderson MM, and Parke HC (1948) Comparative properties of six phenethylamines, with observations on the nature of tachyphylaxis. $J$ Pharmacol Exp Ther 93:63-80.

Wolgin DL (2012) Amphetamine stereotypy, the basal ganglia, and the "selection problem". Behav Brain Res 231:297-308.

Address correspondence to: Charles W. Schindler, Designer Drug Research Unit, NIH/NIDA Intramural Research Program, 251 Bayview Blvd., Baltimore, MD 21224. E-mail: cschind@helix.nih.gov 